\title{
Estimating Average and Local Average Treatment Effects of Education When Compulsory Schooling Laws Really Matter: Corrigendum
}

August, 2008

Philip Oreopoulos

Department of Economics, University of British Columbia philip.oreopoulos@ubc.ca

In the March 2006 edition of this journal, I published an article entitled “Estimating Average and Local Average Treatment Effects of Education when Compulsory School Laws Really Matter” (American Economic Review, Vol. 96, No. 1, March 2006, pp. 152-175). In the last year, some colleagues have informed me that they have had difficulty replicating the UK results using the code I provided in a data appendix. ${ }^{1}$ Through these discussions I learned that a few sampling restrictions that were mentioned in the paper were not in the code, and that some datasets were not merged correctly (for example, individuals were matched based on person and household identifiers, but not family identifiers). The British earnings measure for 1994 was also accidentally dropped. This corrigendum therefore updates the code for producing a revised set of UK results which are qualitatively similar to the original results. The revised output does not affect the discussion or conclusions of the original article.

One of the primary ideas behind the original article is that the remarkably large response from changes to compulsory schooling laws in the UK provides a rare

\footnotetext{
${ }^{1}$ I thank Paul Devereux, Heather Royer, Joseph Shapiro, and Raymond Guiteras for pointing me to these mistakes. I am a strong supporter of making available code for replication purposes, and I am grateful that these errors were identified using the paper's data appendix. Part of the difficulty reconciling the results was due to keeping only an aggregated version of the data. For the revised results here, I include the full micro dataset.
} 
opportunity to measure average returns to schooling for a more general population than compared to previous papers using instrumental variables methodology. First stage estimates suggest that raising the school leaving age from 14 to 15 in 1947 for Great Britain and in 1957 for Northern Ireland affected between 40 to 50 percent of the general cohort population. The original article concluded that the similar UK returns to compulsory schooling estimates compared to those from the U.S. and Canada (with significantly less affected by the policy changes) suggests that the average treatment effect of an additional year of high school is about as large as local average treatment effect estimated for these countries (at least for the particular birth cohorts examined in the study).

Table 1 shows the estimated first stage effects of the policy changes on the number of years of schooling, the reduced form effects of the policy changes on log earnings, and the instrumental variables estimates for the returns to compulsory schooling for Great Britain and the UK. The originally published results are reported in Panel A. Panel B reports the revised results using the full sample of individuals from the 1984 to 1998 British and Northern Irish General Household Surveys, aged 28 to 64, born between 1921 and 1951 (the same sample restrictions reported in the original paper). ${ }^{2}$ The results for Northern Ireland are almost identical. The first stage effects for Great Britain are also about the same, but the returns to schooling estimates are lower - around 7 percent instead of 15 percent. The 95 percent confidence region around these estimates are quite wide, ranging from returns as low as 0 and as high as 15 percent. The revised difference-

\footnotetext{
${ }^{2}$ While the published paper noted that the 1983 GHHS was included for the sample, income that year was recorded as missing. So, in effect, the sample included only the 1984 to 1998 GHHS.
} 
in-difference estimates from combining the Great Britain and Northern Ireland samples are slightly lower, but still above 10 percent, with standard errors around 0.03 .

Table 2 shows the same set of estimates but with three alternative sample specifications that I believe are equally justifiable. Panel A shows results after restricting the sample to individuals that left full-time schooling by age 18 or less, with the logic that since the first stage results suggest that the policy changes affected whether individuals left full time schooling before age 15 but not before age 16 (these effects are shown in the original article and revised set of tables and figures), this alternative restriction may improve precision by dropping individuals not likely impacted by the policy shift. Panel B shows results from dropping individuals aged 61 to 64 . Earnings from older workers close to retirement may be more volatile. Panel C shows results from adding more data using more recent years of the British and Northern Irish General Household Surveys that were not available when I began the study (1999 to 2006).

The resulting first stage effects for Great Britain in Panel A from dropping individuals that left school after age 18 are generally not different from the full sample in Panel B of Table 1, except the first stage effects from the school leaving age change in Northern Ireland are lower, and the instrumental variables estimates have larger standard errors. Dropping individuals over 60 years of age in Panel B generates higher point estimates for the reduced form and returns to compulsory schooling British estimates. The Northern Ireland estimates are generally unchanged. Adding the additional survey data in Panel C notably improves precision and leads to significant returns to education estimates that range from 11 to 13 percent for Great Britain and 13 to 18 percent for Northern Ireland. Finally, Panel D combines all three alternative sample specifications, 
which leads to similar point estimates compared to the original article, but large standard errors for the Northern Ireland sample.

As mentioned in the original article, the regression discontinuity approach leads to greater imprecision than the difference-in-difference approach, given that earnings are tapering off for successively older birth cohorts at the time the discontinuity occurs. Since the confidence intervals include values lower than the point estimates for Canada and the U.S., the analysis requires considering the robustness and general patterns of the results under alternative samples, methodologies, and conditions. In my opinion, the results presented here, the robustness checks presented in this article’s appendix, and the results presented in Oreopoulos (2007) using the Eurobarometer Surveys, point to clear evidence of substantial returns to compulsory schooling - between 8 and 15 percent for individuals affected by these policy changes.

I have created a new set of tables and figures from the specification above that adds additional survey years from the General Household Survey, as shown in Table 2, Panel C). These can be found in the data appendix. Code to replicate these results, along with the full micro dataset, is also provided in the data appendix. The results still support the conclusions and discussion that I drew using tables of the published version.

\section{References}

Oreopoulos, Philip. “Do Dropouts Drop Out Too Soon? Wealth, Health, and Happiness

from Compulsory Schooling,” Journal of Public Economics 2007, 91, (11-12), 22132229. 
Table 1

First Stage, Reduced Form, and IV Estimates for Returns to Compulsory Schooling

Great Britain and Northern Ireland, Baseline Sample

\begin{tabular}{|c|c|c|c|c|c|c|c|c|c|}
\hline & (1) & (2) & (3) & (4) & (5) & (6) & (7) & (8) & (9) \\
\hline & \multicolumn{3}{|c|}{$\begin{array}{c}\text { (First Stage) } \\
\text { Dependent Variable: Age Finished Full Time School }\end{array}$} & \multicolumn{3}{|c|}{$\begin{array}{l}\text { (Reduced Form) } \\
\text { Dependent Variable: Log Annual Earnings }\end{array}$} & \multicolumn{3}{|c|}{$\begin{array}{l}\text { (IV Returns to Compulsory Schooling) } \\
\text { Dependent Variable: Log Annual Earnings }\end{array}$} \\
\hline & \multicolumn{9}{|c|}{ Panel A: Published Results } \\
\hline $\begin{array}{l}\text { Great Britain } \\
\text { [n=57624] }\end{array}$ & $\begin{array}{c}0.440 \\
{[0.065]^{* * *}}\end{array}$ & $\begin{array}{c}0.436 \\
{[0.071]^{* * *}}\end{array}$ & $\begin{array}{c}0.453 \\
{[0.076]^{* * *}}\end{array}$ & $\begin{array}{c}0.065 \\
{[0.025]^{* *}}\end{array}$ & $\begin{array}{c}0.064 \\
{[0.026]^{* *}}\end{array}$ & $\begin{array}{c}0.042 \\
{[0.043]}\end{array}$ & $\begin{array}{c}0.147 \\
{[0.061]^{* *}}\end{array}$ & $\begin{array}{c}0.145 \\
{[0.063]^{* *}}\end{array}$ & $\begin{array}{c}0.149 \\
{[0.064]^{* *}}\end{array}$ \\
\hline $\begin{array}{l}\text { Northern Ireland } \\
\text { [ } \mathrm{n}=8921]\end{array}$ & $\begin{array}{c}0.397 \\
{[0.074]^{* * *}}\end{array}$ & $\begin{array}{c}0.391 \\
{[0.073]^{* * *}}\end{array}$ & $\begin{array}{c}0.353 \\
{[0.100]^{* * *}}\end{array}$ & $\begin{array}{c}0.054 \\
{[0.027]^{*}}\end{array}$ & $\begin{array}{c}0.074 \\
{[0.025]^{* * *}}\end{array}$ & $\begin{array}{c}0.074 \\
{[0.045]}\end{array}$ & $\begin{array}{c}0.135 \\
{[0.071]^{*}}\end{array}$ & $\begin{array}{c}0.187 \\
{[0.070]^{* *}}\end{array}$ & $\begin{array}{c}0.21 \\
{[0.135]}\end{array}$ \\
\hline \multirow[t]{2}{*}{$\begin{array}{l}\text { G. Britain and N. Ireland } \\
\text { with N. Ireland Fixed Effect } \\
\text { [n=66185] }\end{array}$} & $\begin{array}{c}0.418 \\
{[0.040]^{* * *}}\end{array}$ & $\begin{array}{c}0.397 \\
{[0.043]^{* * *}}\end{array}$ & $\begin{array}{c}0.401 \\
{[0.045]^{* * *}}\end{array}$ & $\begin{array}{c}0.073 \\
{[0.016]^{* * *}}\end{array}$ & $\begin{array}{c}0.058 \\
{[0.016]^{* * *}}\end{array}$ & $\begin{array}{c}0.059 \\
{[0.018]^{* * *}}\end{array}$ & $\begin{array}{c}0.174 \\
{[0.042]^{* * *}}\end{array}$ & $\begin{array}{c}0.149 \\
{[0.044]^{* * *}}\end{array}$ & $\begin{array}{c}0.148 \\
{[0.046]^{* * *}}\end{array}$ \\
\hline & \multicolumn{9}{|c|}{ Panel B: Revised Results with Baseline Sample: 1921 - 1951 Birth Cohorts aged 28-64 in the 1984 - 1998 GHHS } \\
\hline $\begin{array}{l}\text { Great Britain } \\
{[\mathrm{n}=55088]}\end{array}$ & $\begin{array}{c}0.408 \\
{[0.063]^{* * *}}\end{array}$ & $\begin{array}{c}0.408 \\
{[0.064]^{* * *}}\end{array}$ & $\begin{array}{c}0.435 \\
{[0.073]^{* * *}}\end{array}$ & $\begin{array}{c}0.029 \\
{[0.016]^{*}}\end{array}$ & $\begin{array}{c}0.025 \\
{[0.020]}\end{array}$ & $\begin{array}{c}0.032 \\
{[0.021]}\end{array}$ & $\begin{array}{c}0.069 \\
{[0.040]^{*}}\end{array}$ & $\begin{array}{c}0.066 \\
{[0.050]}\end{array}$ & $\begin{array}{c}0.067 \\
{[0.049]}\end{array}$ \\
\hline $\begin{array}{l}\text { Northern Ireland } \\
\mathrm{n}=[8954]\end{array}$ & $\begin{array}{c}0.456 \\
{[0.104]^{* * *}}\end{array}$ & $\begin{array}{c}0.444 \\
{[0.105]^{* * *}}\end{array}$ & $\begin{array}{c}0.413 \\
{[0.082]^{* * *}}\end{array}$ & $\begin{array}{c}0.059 \\
{[0.040]}\end{array}$ & $\begin{array}{c}0.081 \\
{[0.034]^{* *}}\end{array}$ & $\begin{array}{c}0.074 \\
{[0.045]}\end{array}$ & $\begin{array}{c}0.129 \\
{[0.076]^{*}}\end{array}$ & $\begin{array}{c}0.18 \\
{[0.062]^{* * *}}\end{array}$ & $\begin{array}{c}0.179 \\
{[0.096]^{*}}\end{array}$ \\
\hline $\begin{array}{l}\text { G. Britain and N. Ireland } \\
\text { with N. Ireland Fixed Effect } \\
\text { [n=64042] }\end{array}$ & $\begin{array}{c}0.437 \\
{[0.043]^{* * *}}\end{array}$ & $\begin{array}{c}0.44 \\
{[0.044]^{* * *}}\end{array}$ & $\begin{array}{c}0.451 \\
{[0.044]^{* * *}}\end{array}$ & $\begin{array}{c}0.058 \\
{[0.013]^{* * *}}\end{array}$ & $\begin{array}{c}0.045 \\
{[0.014]^{* * *}}\end{array}$ & $\begin{array}{c}0.044 \\
{[0.014]^{* * *}}\end{array}$ & $\begin{array}{c}0.132 \\
{[0.031]^{* * *}}\end{array}$ & $\begin{array}{c}0.105 \\
{[0.030]^{* * *}}\end{array}$ & $\begin{array}{c}0.097 \\
{[0.030]^{* * *}}\end{array}$ \\
\hline $\begin{array}{l}\text { Birth Cohort } \\
\text { Polynomial Controls }\end{array}$ & Quartic & Quartic & Quartic & Quartic & Quartic & Quartic & Quartic & Quartic & Quartic \\
\hline Age Polynomial Controls & None & Quartic & None & None & Quartic & None & None & Quartic & None \\
\hline Age Dummies & No & No & Yes & No & No & Yes & No & No & Yes \\
\hline
\end{tabular}

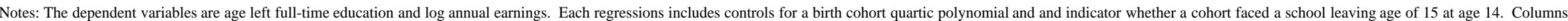

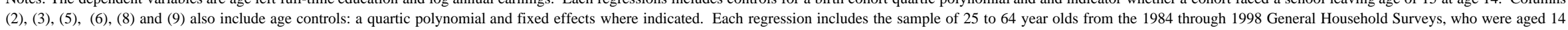
between 1935 and 1965. Data are first aggregated into cell means and weighted by cell size. Regressions are clustered by birth cohort and region (Britian or N. Ireland). [n $=$ sample size] 
Table 2

First Stage, Reduced Form, and IV Estimates for Returns to Compulsory Schooling

\begin{tabular}{|c|c|c|c|c|c|c|c|c|c|}
\hline \multirow[b]{4}{*}{$\begin{array}{l}\text { Great Britain } \\
{[\mathrm{n}=46760]}\end{array}$} & \multirow{2}{*}{\multicolumn{3}{|c|}{$\begin{array}{r}\text { (3) } \\
\text { Ill Time School }\end{array}$}} & (4) & (5) & (6) & (7) & (8) & (9) \\
\hline & & & & \multicolumn{3}{|c|}{$\begin{array}{l}\text { (Reduced Form) } \\
\text { Dependent Variable: Log Annual Earnings }\end{array}$} & \multicolumn{3}{|c|}{$\begin{array}{l}\text { (IV Returns to Compulsory Schooling) } \\
\text { Dependent Variable: Log Annual Earnings }\end{array}$} \\
\hline & \multicolumn{9}{|c|}{ Panel A: 1921 - 1951 Birth Cohorts aged $28-64$ in the 1984 - 1998 GHHS, left full time schooling < 19} \\
\hline & $\begin{array}{l}0.437 \\
{[0.080]^{* * *}}\end{array}$ & $\begin{array}{c}0.438 \\
{[0.079] * * *}\end{array}$ & $\begin{array}{c}0.44 \\
{[0.078]^{* * * *}}\end{array}$ & $\begin{array}{c}0.032 \\
{[0.015]^{* * *}}\end{array}$ & $\begin{array}{c}0.029 \\
{[0.019]}\end{array}$ & $\begin{array}{c}0.037 \\
{[0.020]^{*}}\end{array}$ & $\begin{array}{c}0.073 \\
{[0.042]^{*}}\end{array}$ & $\begin{array}{c}0.062 \\
{[0.050]}\end{array}$ & $\begin{array}{c}0.084 \\
{[0.053]}\end{array}$ \\
\hline $\begin{array}{l}\text { Northern Ireland } \\
{[\mathrm{n}=7676]}\end{array}$ & $\begin{array}{c}0.24 \\
{[0.068]^{* * * *}}\end{array}$ & $\begin{array}{l}0.24 \\
{[0.068]^{* * * *}}\end{array}$ & $\begin{array}{l}0.274 \\
{[0.072]^{* * * *}}\end{array}$ & $\begin{array}{c}0.03 \\
{[0.046]}\end{array}$ & $\begin{array}{c}0.054 \\
{[0.037]}\end{array}$ & $\begin{array}{c}0.059 \\
{[0.048]}\end{array}$ & $\begin{array}{c}0.123 \\
{[0.194]}\end{array}$ & $\begin{array}{c}0.224 \\
{[0.155]}\end{array}$ & $\begin{array}{c}0.203 \\
{[0.199]}\end{array}$ \\
\hline \multirow[t]{2}{*}{$\begin{array}{l}\text { G. Britain and N. Irelanc } \\
\text { with N. Ireland Fixed Effec } \\
\text { [n=54436] }\end{array}$} & $\begin{array}{c}0.478 \\
{[0.045] * * *}\end{array}$ & $\begin{array}{c}0.477 \\
{[0.045]^{* * *}}\end{array}$ & $\begin{array}{c}0.48 \\
{[0.043]^{* * * *}}\end{array}$ & $\begin{array}{c}0.058 \\
{[0.013]^{* * *}}\end{array}$ & $\begin{array}{c}0.044 \\
{[0.013]^{* * *}}\end{array}$ & $\begin{array}{c}0.043 \\
{[0.014]^{* * *}}\end{array}$ & $\begin{array}{c}0.123 \\
{[0.030]^{* * *}}\end{array}$ & $\begin{array}{l}0.091 \\
{[0.031]^{* * * *}}\end{array}$ & $\begin{array}{c}0.091 \\
{[0.032]^{* * * *}}\end{array}$ \\
\hline & \multicolumn{9}{|c|}{ Panel B: 1921 - 1951 Birth Cohorts aged 28-60 in the 1984 - 1998 GHHS } \\
\hline $\begin{array}{l}\text { Great Britain } \\
{[\mathrm{n}=51643]}\end{array}$ & $\begin{array}{c}0.376 \\
{[0.054]^{* * *}}\end{array}$ & $\begin{array}{c}0.376 \\
{[0.053]^{* * *}}\end{array}$ & $\begin{array}{c}0.411 \\
{[0.063]^{* * * *}}\end{array}$ & $\begin{array}{c}0.047 \\
{[0.020]^{* * *}}\end{array}$ & $\begin{array}{c}0.044 \\
{[0.022]^{*}}\end{array}$ & $\begin{array}{c}0.052 \\
{[0.023]^{* *}}\end{array}$ & $\begin{array}{c}0.108 \\
{[0.057]^{*}}\end{array}$ & $\begin{array}{c}0.144 \\
{[0.079]^{*}}\end{array}$ & $\begin{array}{c}0.107 \\
{[0.062]^{*}}\end{array}$ \\
\hline $\begin{array}{l}\text { Northern Ireland } \\
{[\mathrm{n}=8311]}\end{array}$ & $\begin{array}{c}0.434 \\
{[0.107] * * *}\end{array}$ & $\begin{array}{c}0.436 \\
{[0.110] * * *}\end{array}$ & $\begin{array}{c}0.372 \\
{[0.094]^{* * *}}\end{array}$ & $\begin{array}{c}0.051 \\
{[0.044]}\end{array}$ & $\begin{array}{c}0.085 \\
{[0.036]^{* *}}\end{array}$ & $\begin{array}{c}0.083 \\
{[0.046]^{*}}\end{array}$ & $\begin{array}{c}0.094 \\
{[0.097]}\end{array}$ & $\begin{array}{c}0.179 \\
{[0.064]^{* *}}\end{array}$ & $\begin{array}{c}0.226 \\
{[0.098]^{* *}}\end{array}$ \\
\hline \multirow[t]{2}{*}{$\begin{array}{l}\text { G. Britain and N. Irelanc } \\
\text { with N. Ireland Fixed Effec } \\
{[\mathrm{n}=59954]}\end{array}$} & $\begin{array}{c}0.411 \\
{[0.043]^{* * *}}\end{array}$ & $\begin{array}{c}0.41 \\
{[0.042]^{* * *}}\end{array}$ & $\begin{array}{c}0.425 \\
{[0.042]^{* * *}}\end{array}$ & $\begin{array}{c}0.064 \\
{[0.014]^{* * *}}\end{array}$ & $\begin{array}{c}0.046 \\
{[0.014]^{* * * *}}\end{array}$ & $\begin{array}{c}0.046 \\
{[0.014] * * *}\end{array}$ & $\begin{array}{c}0.159 \\
{[0.038]^{* * *}}\end{array}$ & $\begin{array}{c}0.112 \\
{[0.037]^{* * *}}\end{array}$ & $\begin{array}{c}0.11 \\
{[0.037]^{* * *}}\end{array}$ \\
\hline & \multicolumn{9}{|c|}{ Panel C: 1921 - 1951 Birth Cohorts aged 28-64 in the 1984 - 2006 GHHS } \\
\hline $\begin{array}{l}\text { Great Britain } \\
\text { [n=73954] }\end{array}$ & $\begin{array}{l}0.495 \\
{[0.074]^{* * *}}\end{array}$ & $\begin{array}{l}0.457 \\
{[0.065]^{* * * *}}\end{array}$ & $\begin{array}{l}0.472 \\
{[0.069]^{* * * *}}\end{array}$ & $\begin{array}{l}0.055 \\
{[0.015] * * *}\end{array}$ & $\begin{array}{c}0.052 \\
{[0.014]^{* * * *}}\end{array}$ & $\begin{array}{l}0.056 \\
{[0.017]^{* * * *}}\end{array}$ & $\begin{array}{l}0.112 \\
{[0.034]^{* * * *}}\end{array}$ & $\begin{array}{l}0.111 \\
{[0.033]^{* * * *}}\end{array}$ & $\begin{array}{c}0.125 \\
{[0.040] * * *}\end{array}$ \\
\hline $\begin{array}{l}\text { Northern Ireland } \\
{[\mathrm{n}=8954]}\end{array}$ & $\begin{array}{c}0.456 \\
{[0.104]^{* * * *}}\end{array}$ & $\begin{array}{c}0.444 \\
{[0.105]^{* * * *}}\end{array}$ & $\begin{array}{c}0.413 \\
{[0.082]^{* * * *}}\end{array}$ & $\begin{array}{c}0.059 \\
{[0.040]}\end{array}$ & $\begin{array}{c}0.081 \\
{[0.034]^{* *}}\end{array}$ & $\begin{array}{c}0.074 \\
{[0.045]}\end{array}$ & $\begin{array}{c}0.129 \\
{[0.076]^{*}}\end{array}$ & $\begin{array}{c}0.18 \\
{[0.062]^{* * * *}}\end{array}$ & $\begin{array}{c}0.179 \\
{[0.096]^{*}}\end{array}$ \\
\hline \multirow[t]{2}{*}{$\begin{array}{l}\text { G. Britain and N. Irelanc } \\
\text { with N. Ireland Fixed Effec } \\
{[n=82908]}\end{array}$} & $\begin{array}{c}0.491 \\
{[0.042] * * *}\end{array}$ & $\begin{array}{c}0.475 \\
[0.044]]^{* * *}\end{array}$ & $\begin{array}{c}0.485 \\
{[0.042]^{* * * *}}\end{array}$ & $\begin{array}{c}0.02 \\
{[0.015]}\end{array}$ & $\begin{array}{c}0.065 \\
{[0.013]^{* * * *}}\end{array}$ & $\begin{array}{c}0.065 \\
{[0.013]^{* * * *}}\end{array}$ & $\begin{array}{c}0.041 \\
{[0.032]}\end{array}$ & $\begin{array}{c}0.133 \\
{[0.027]^{* * * *}}\end{array}$ & $\begin{array}{c}0.135 \\
{[0.028]^{* * *}}\end{array}$ \\
\hline & \multicolumn{9}{|c|}{ Panel D: 1921 - 1951 Birth Cohorts aged 28-60 in the 1979 - $2006 \mathrm{GHHS}$, left full time schooling < 19} \\
\hline $\begin{array}{l}\text { Great Britain } \\
\text { [n=54982] }\end{array}$ & $\begin{array}{c}0.428 \\
{[0.086] * * *}\end{array}$ & $\begin{array}{c}0.428 \\
{[0.086]^{* * * *}}\end{array}$ & $\begin{array}{c}0.431 \\
{[0.085]^{* * * *}}\end{array}$ & $\begin{array}{c}0.063 \\
{[0.018]^{* * * *}}\end{array}$ & $\begin{array}{c}0.047 \\
{[0.023]^{* *}}\end{array}$ & $\begin{array}{c}0.052 \\
{[0.024]^{* *}}\end{array}$ & $\begin{array}{c}0.191 \\
{[0.074]^{* *}}\end{array}$ & $\begin{array}{c}0.118 \\
{[0.073]}\end{array}$ & $\begin{array}{c}0.133 \\
{[0.076]^{*}}\end{array}$ \\
\hline $\begin{array}{l}\text { Northern Ireland } \\
{[\mathrm{n}=7081]}\end{array}$ & $\begin{array}{c}0.199 \\
{[0.054]^{* * * *}}\end{array}$ & $\begin{array}{c}0.209 \\
{[0.056] * * *}\end{array}$ & $\begin{array}{c}0.227 \\
{[0.055]^{* * * *}}\end{array}$ & $\begin{array}{c}0.022 \\
{[0.048]}\end{array}$ & $\begin{array}{c}0.057 \\
{[0.036]}\end{array}$ & $\begin{array}{c}0.068 \\
{[0.048]}\end{array}$ & $\begin{array}{c}0.168 \\
{[0.204]}\end{array}$ & $\begin{array}{c}0.313 \\
{[0.127] * *}\end{array}$ & $\begin{array}{c}0.311 \\
{[0.184]}\end{array}$ \\
\hline $\begin{array}{l}\text { G. Britain and N. Irelani } \\
\text { with N. Ireland Fixed Effec } \\
{[\mathrm{n}=62063]}\end{array}$ & $\begin{array}{c}0.474 \\
{[0.047] * * *}\end{array}$ & $\begin{array}{c}0.481 \\
{[0.048]^{* * * *}}\end{array}$ & $\begin{array}{c}0.483 \\
[0.046]]^{* * * *}\end{array}$ & $\begin{array}{c}0.015 \\
{[0.018]}\end{array}$ & $\begin{array}{c}0.062 \\
{[0.015]^{* * * *}}\end{array}$ & $\begin{array}{c}0.062 \\
{[0.014]^{* * * *}}\end{array}$ & $\begin{array}{c}0.035 \\
{[0.040]}\end{array}$ & $\begin{array}{c}0.131 \\
{[0.036]^{* * * *}}\end{array}$ & $\begin{array}{c}0.127 \\
{[0.035]^{* * *}}\end{array}$ \\
\hline $\begin{array}{l}\text { Birth Cohort } \\
\text { Polynomial Controls }\end{array}$ & Quartic & Quartic & Quartic & Quartic & Quartic & Quartic & Quartic & Quartic & Quartic \\
\hline Age Polynomial Controls & None & Quartic & None & None & Quartic & None & None & Quartic & None \\
\hline Age Dummies & No & No & Yes & No & No & Yes & No & No & Yes \\
\hline
\end{tabular}

Notes: The dependent variables are age left full-time education and log annual earnings. Each regressions includes controls for a birth cohort quartic polynomial and and indicator whether a cohort faced a school leaving age of 15 at age 14 . Columns (2), (3), (5), (6), (8) and (9) also include age controls: a quartic polynomial and fixed effects where indicated. Each regression includes the sample from the 1984 through 1998 General Household Surveys, who were aged 14 between 1935 and 1965 . Data are
first aggregated into cell means and weighted by cell size. Regressions are clustered by birth cohort and region (Britian or N. Ireland). [n s sample size] 